\title{
Mutual Coupling Effect on Classical Three-way Doherty Amplifier for 5G Applications
}

\author{
Ahmed M. Abdulkhaleq ${ }^{1,2}$, Khalid W Hameed ${ }^{2}$, Maan A. Yahya ${ }^{3}$, Yasir Al-Yasir ${ }^{2}$, Naser \\ Ojaroudi Parchin ${ }^{2}$, Syed M. H. Syed Anera ${ }^{1}$, Ashwain Rayit ${ }^{1}$, and Raed A. Abd-Alhameed ${ }^{2}$ \\ \{a.abd@sarastech.co.uk\} \\ ${ }^{1}$ SARAS Technology, Leeds, UK \\ ${ }^{2}$ School of Engineering, Design and Technology, University of Bradford, Bradford, UK \\ ${ }^{3}$ Ninevah Technical Institute, Northern Technical University, Mosul, Iraq

\begin{abstract}
A three-way Doherty power amplifier targeting sub 6-GHz. Three 6W GaN transistors are used to design an $18 \mathrm{~W}$ peak power with an $8.5 \mathrm{~dB}$ back-off efficiency. The amplifier achieved an efficiency of $55 \%, 63 \%$ and $80 \%$ at $8.5,6$, and $0 \mathrm{~dB}$ respectively from the peak power. The designed amplifier target 3.4-3.8 GHz band with a gain of 13 $\mathrm{dB}$ over the band. In addition, the amplifier was examined for different VSWRs taking into account the effect of impedance changing of antenna due to beamforming. There was an average of $3.35 \mathrm{dBm}$ variation in the output power and $20 \%$ efficiency variation for different VSWRs at both the back-off and peaking regions. The structure of three-way Doherty amplifier or sequence of turning the peaking amplifiers on affects the amplifier performance for different VSWRs.
\end{abstract}

Keywords: Efficiency; Beamforming; Antenna; Three-way Doherty amplifier; VSWR; output power.

\section{Introduction}

The wireless mobile communications systems are utilising modulation schemes with high complexity to increase the data rate and to employ the allocated bandwidth effectively due to including additional services and features that attract the user; however, the modulated signal will have an envelope which can be measured by the peak-to-average-power ratio(PAPR) [1-5]. The efficiency of power amplifiers can be achieved at the saturation region. Nevertheless, for modulated signals with high PAPR, power amplifiers should be operated at the backed-off region that is far from the most efficient point to keep the required linearity performance, however, the efficiency drops sharply [3-11]. The back-off efficiency of power amplifier can be improved using several techniques; among them, the Doherty technique is the easiest, where no digital signal process (DSP) nor additional complex circuity is required. However, this technique suffers from bandwidth limitation, due to the transistor type, matching network design and the impedance inverter. The semiconductor's layers and fabrication methods determine the achievable power and bandwidth, where the bandwidth is inversely proportional to the parasitic capacitance of the drain side. Moreover, the matching networks of the amplifier can be divided into two types: the input matching network which is responsible for controlling the gain over the designed band in addition to the gain flatness, whereas the output matching network is responsible for specifying the achievable power and efficiency [7]. As transistor power capability 
increases, the optimum load impedance that needs to be matched at the output side will be decreased; in this case, the impedance transformation ratio will be increased. In addition, the transistor parasitic will increase the design complexity, especially at high-frequency design. The third factor that limits the bandwidth of the Doherty amplifier is the impedance invertor network, where it can work perfectly for a single frequency design, but as the design bandwidth increases, the impedance inverter network effect will be increased.

Moreover, the optimum load impedance changing effect was explored in this paper where different Voltage Standing Wave Ratio (VSWR) were applied to the designed amplifier.

\section{Overview of Doherty Amplifier}

The Doherty amplifier operation is explained in detail in many materials; however, a short summary will be provided. W. Doherty, in 1936, came up with a new concept for combining two amplifiers outputs [12]. Doherty used a quarter-wavelength transmission line and two tube amplifiers. Where the first one (carrier amplifier) operates all the time on the other hand, the second amplifier (peaking amplifier) operates only during the load modulation region, at the same time, the bias condition of the carrier amplifier was as class $\mathrm{AB}$, whereas the bias condition of the other amplifier was as class $\mathrm{C}$ amplifier [6]. For the impedance inverter, a quarter wavelength was used as an to invert the impedance that is seen by the carrier amplifier as illustrated in Figure 1.

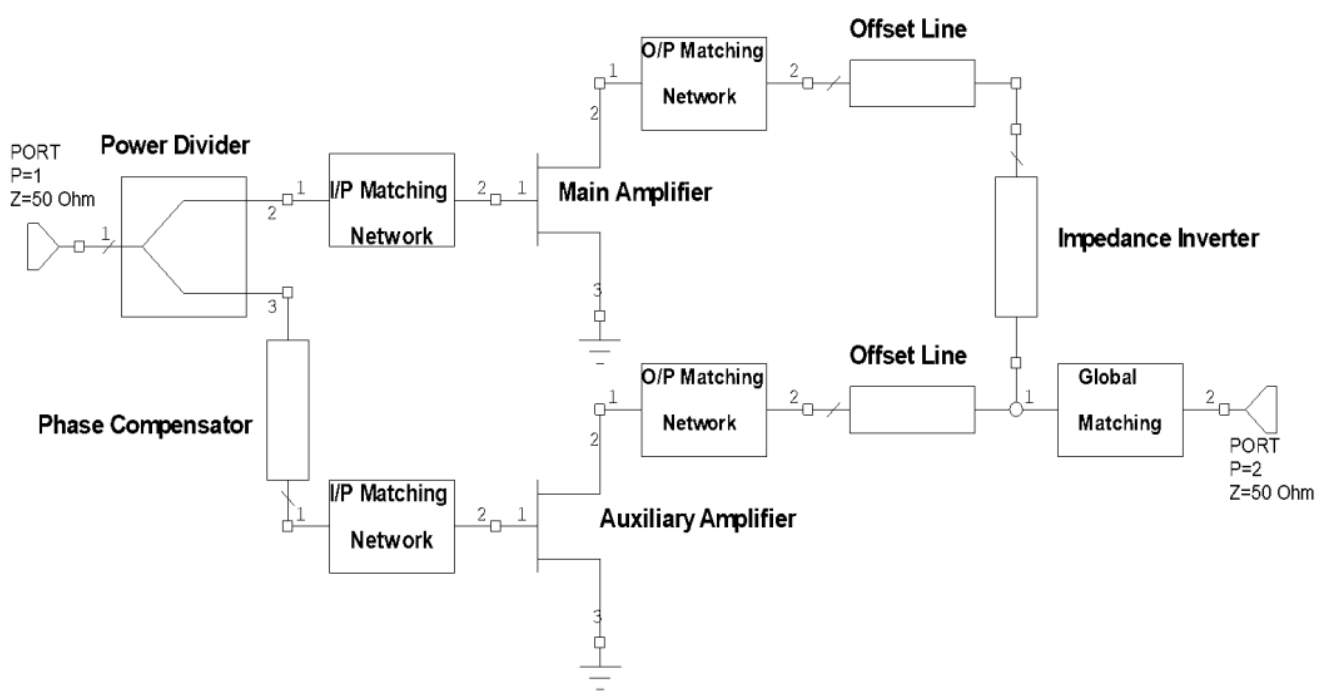

Fig.1 Doherty amplifier structure [7].

The characteristic of a transistor can be explored by applying different gate voltages and checking the behaviour of the drain current, as illustrated in Figure 2. The power amplifier load impedance can be determined using equation (1) assuming that the drain parasitic of the transistor is disregarded:

$$
R_{\text {opt }}=2 \frac{V_{d d}-V_{\text {knee }}}{I_{\max }}
$$




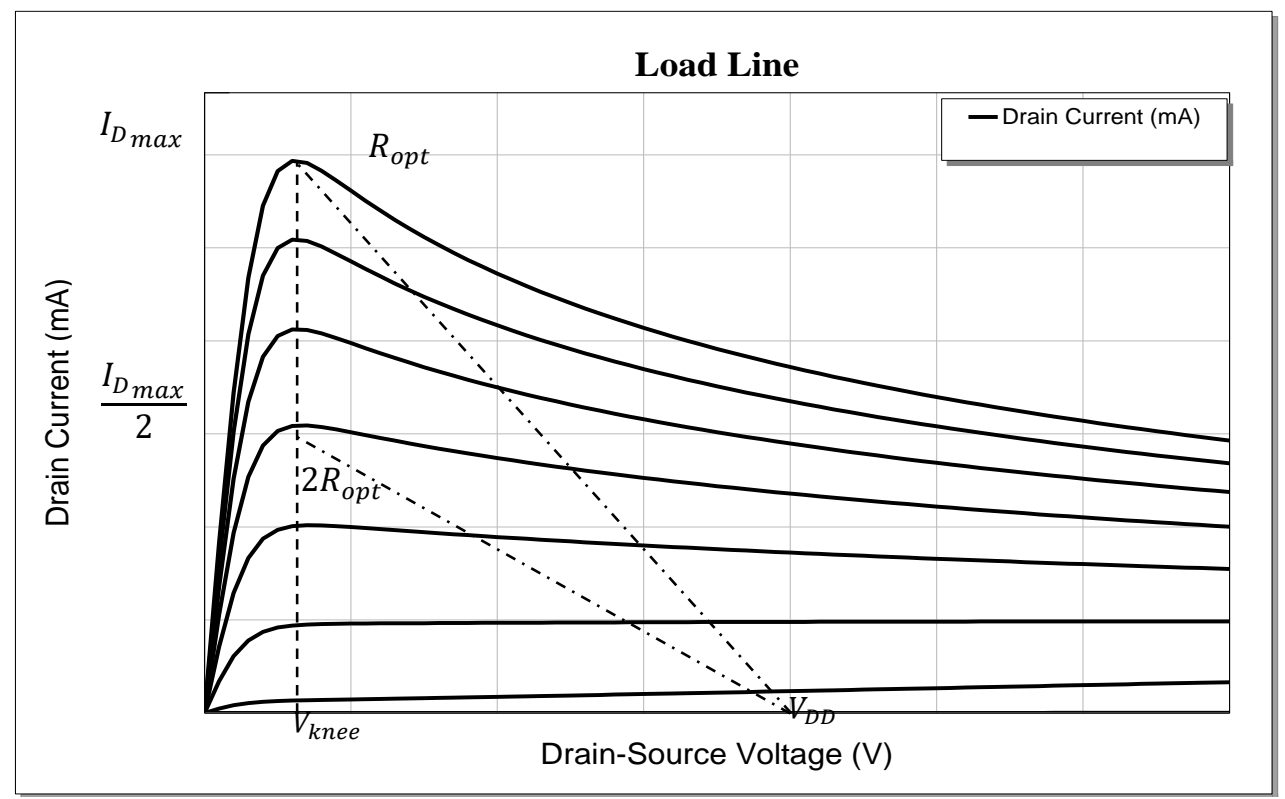

Fig. 2. IV-curves of a transistor [7].

The Doherty amplifier relies on load modulation, where its operations can be summarized by two regions of operation assuming both amplifiers are are connected to a load of $25 \Omega$ and matched to $50 \Omega$.

At low input power region, the carrier amplifier is working, nevertheless, because of the quarter wavelength impedance transmission line inversion property, the impedance seen by the carrier amplifier is $100 \Omega$, which is double the optimum load seen by the carrier amplifier, so the carrier amplifier will be almost saturated, where the first peak efficiency will be seen.

At the load modulation region, the Doherty operation will be clear, where a current will be injected, to the summing node, by the peaking amplifier, so two current will be contributed to the same load. There will be a reduction from a $100 \Omega$ to $50 \Omega$ of the impedance seen by the carrier amplifier depending peaking amplifier current. However, the saturation of the carrier amplifier continues until its maximum power.

\section{Three-way Design of Doherty Amplifier}

Only 6-dB a peak efficiency at the back-off can be obtained from classical Doherty design assuming both amplifiers have same capabilities. To get more efficiency at further back-off of Doherty power, two different capabilities of power transistors of can be used to design asymmetrical Doherty amplifier to accomplish a higher back-off [13-16]. Another way to increase the back-off efficiency operating region is to utilise more parallel transistors connected via more than one quarter-wavelength line as demonstrated figure 3 . Where one of the transistor acts as the main amplifier and the other two amplifiers act as peaking ones. The main amplifier is responsible for the the first peaking efficiency since it is the only working amplifier and other amplifiers are off. Then, a current will be injected by the first peaking amplifier into the load, where the impedance seen by the main amplifier will be changed. Moreover, additional current 
will be contributed to the output power from the other peaking amplifier in the next region as illustrated in Figure4. Equations (2) to (4) represents the currents amount of each amplifier:

$$
\begin{gathered}
I_{\text {main }}= \begin{cases}I_{m} \frac{V_{\text {in }}}{T_{1} V_{\text {max }}} & 0<\frac{v_{\text {in }}}{V_{\text {max }}}<T_{1} \\
I_{m} & T_{1}<\frac{v_{\text {in }}}{V_{\text {max }}}<1\end{cases} \\
I_{\text {Peak } 1}= \begin{cases}0 & 0<\frac{v_{\text {in }}}{V_{\max }}<T_{1} \\
\frac{I_{p 1}}{1-T_{1}}\left(\frac{v_{\text {in }}}{V_{\max }}-T_{1}\right) & T_{1}<\frac{v_{\text {in }}}{V_{\max }}<1\end{cases} \\
I_{\text {Peak2 }}= \begin{cases}0 & 0<\frac{v_{\text {in }}}{V_{\max }}<T_{2} \\
\frac{I_{p 2}}{1-T_{2}}\left(\frac{v_{\text {in }}}{V_{\text {max }}}-T_{2}\right) & T_{2}<\frac{v_{\text {in }}}{V_{\text {max }}}<1\end{cases}
\end{gathered}
$$

Where

$T_{1}<T_{2}$

$I_{m}:$ maximum current of the main amplifier

$I_{p_{1}}$ : maximum current of the first peaking amplifier

$I_{p 2}$ : maximum current of the second peaking amplifier

$T_{1}$ : injecting starting point of the first peaking amplifier

$T_{2}$ : injecting starting point of the second peaking amplifier

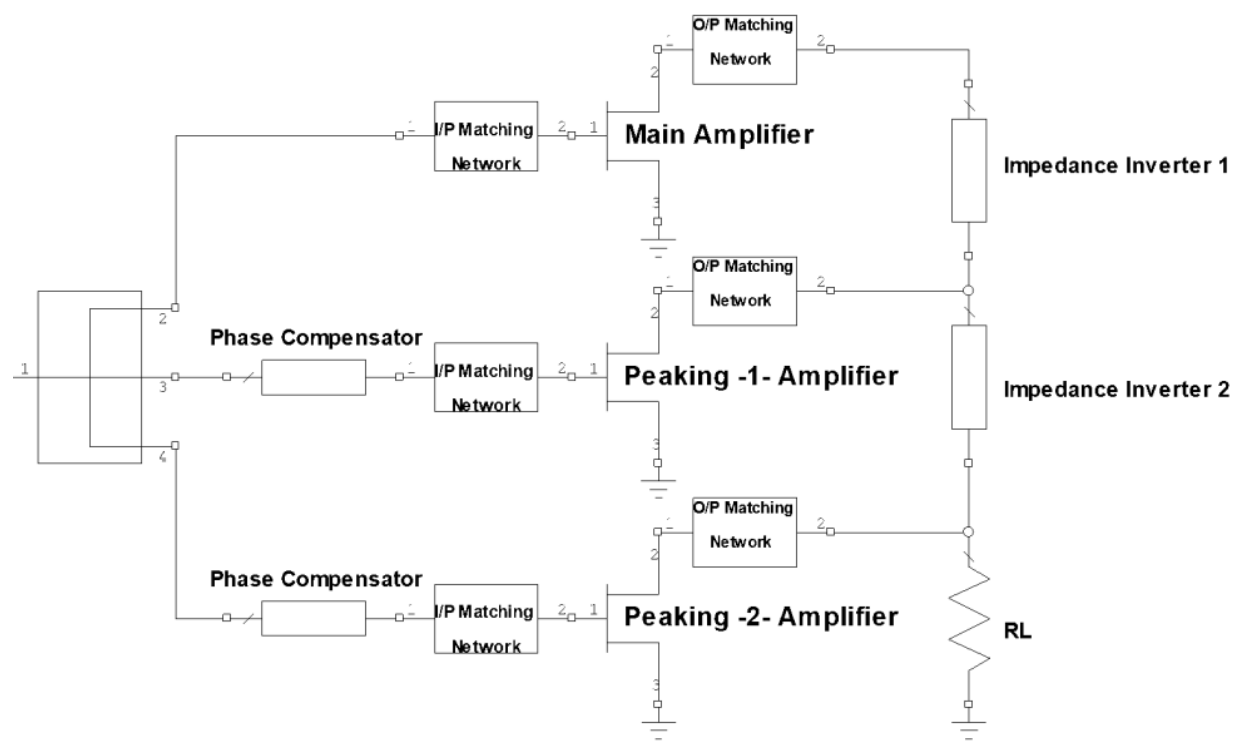

Fig.3. Three-way Doherty circuit layout. 


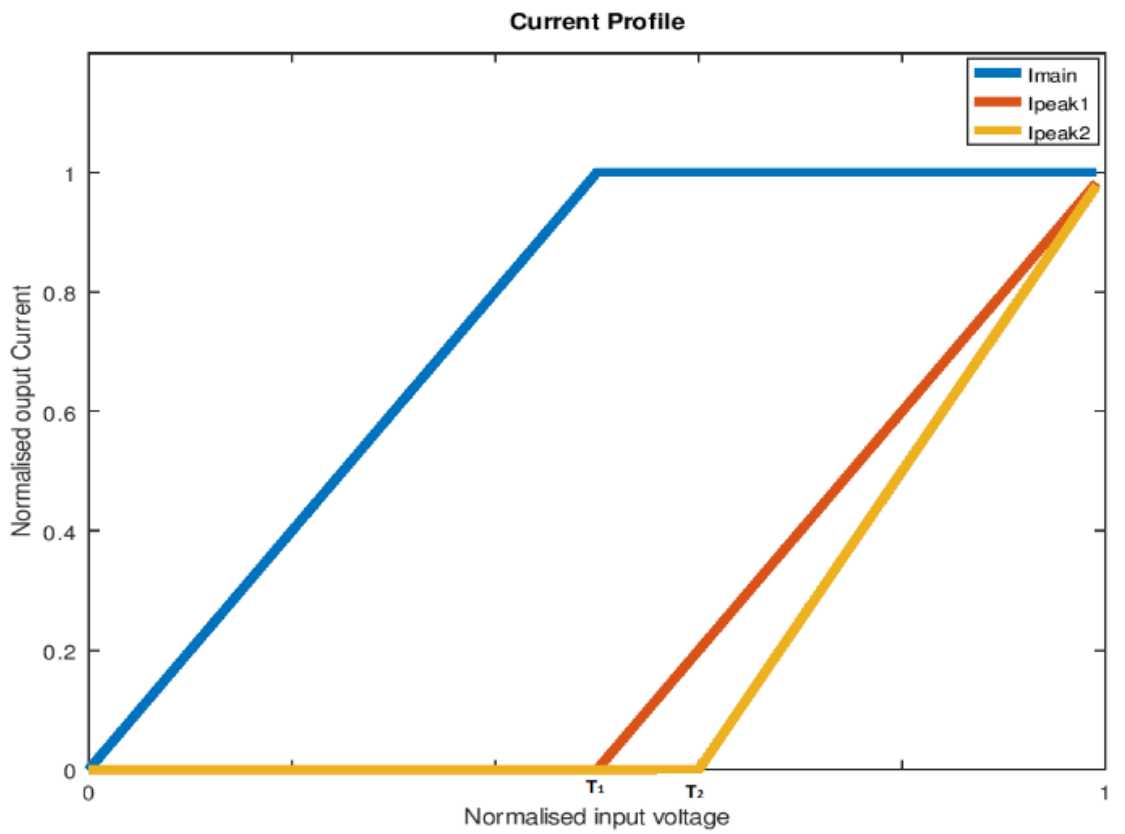

Fig. 4. Three-way Doherty power amplifier current profile.

The designed three-way Doherty performance showed an $8.5 \mathrm{~dB}$ output back-off efficiency with about $55 \%$ drain efficiency, with a linear gain of $13 \mathrm{~dB}$ as illustrated in figure 5 . Moreover, a $43.2 \mathrm{dBm}$ of peak power was achieved with a drain efficiency of over $80 \%$.

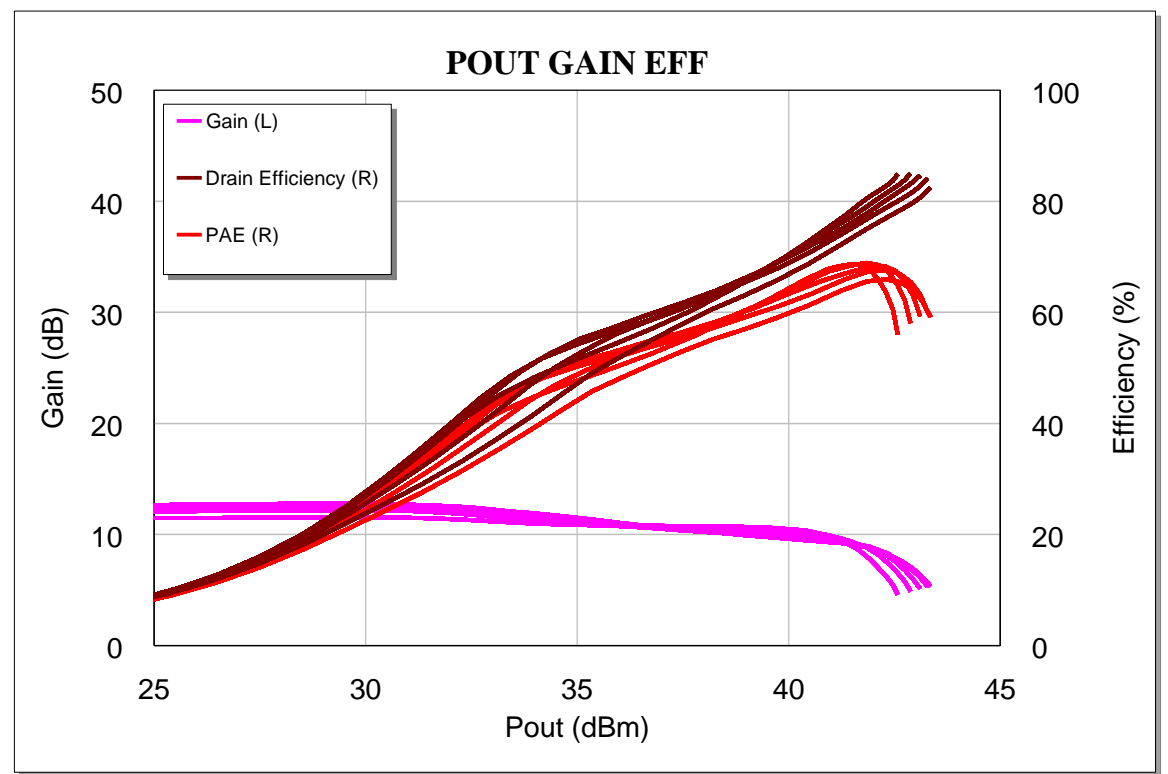

Fig. 5. Gain and drain effeicncy performance of three-way Doherty amplifier. 
The Microwave Office (MWO) was used to simulate the performance of the designed amplifier, where the designed amplifier is matched to the common impedance $(50 \Omega)$; nevertheless, when beamforming technique and MIMO are applied, the antennas impedance varies. Figure 6 shows the selected Voltage Standing Wave Ratio (VSWRs), where the designed three-way amplifier performance is checked after forcing the amplifier to see these impedances due to the change of the VSWRs, Figure 7 and Figure 8 show the output power variation of the designed amplifier at the back-off region and the peak power region respectively. In addition, for the same tested regions, Figure 9 and Figure 10 showed the variations of the efficiency. Due to the impedance change, the amplifier performance is changed significantly. The maximum variation of the power was $3.5 \mathrm{dBm}$ at the peak power and $3.2 \mathrm{dBm}$ at the back-off region, whereas $21.3 \%$ was the efficiency variation at the peak power and $17.8 \%$ at the back-off power. This performance can be compared with [8], where more variation in the power was obtained and less variation in the efficiency, so that, the amplifier structure has a significant effect when different VSWRs are applied.

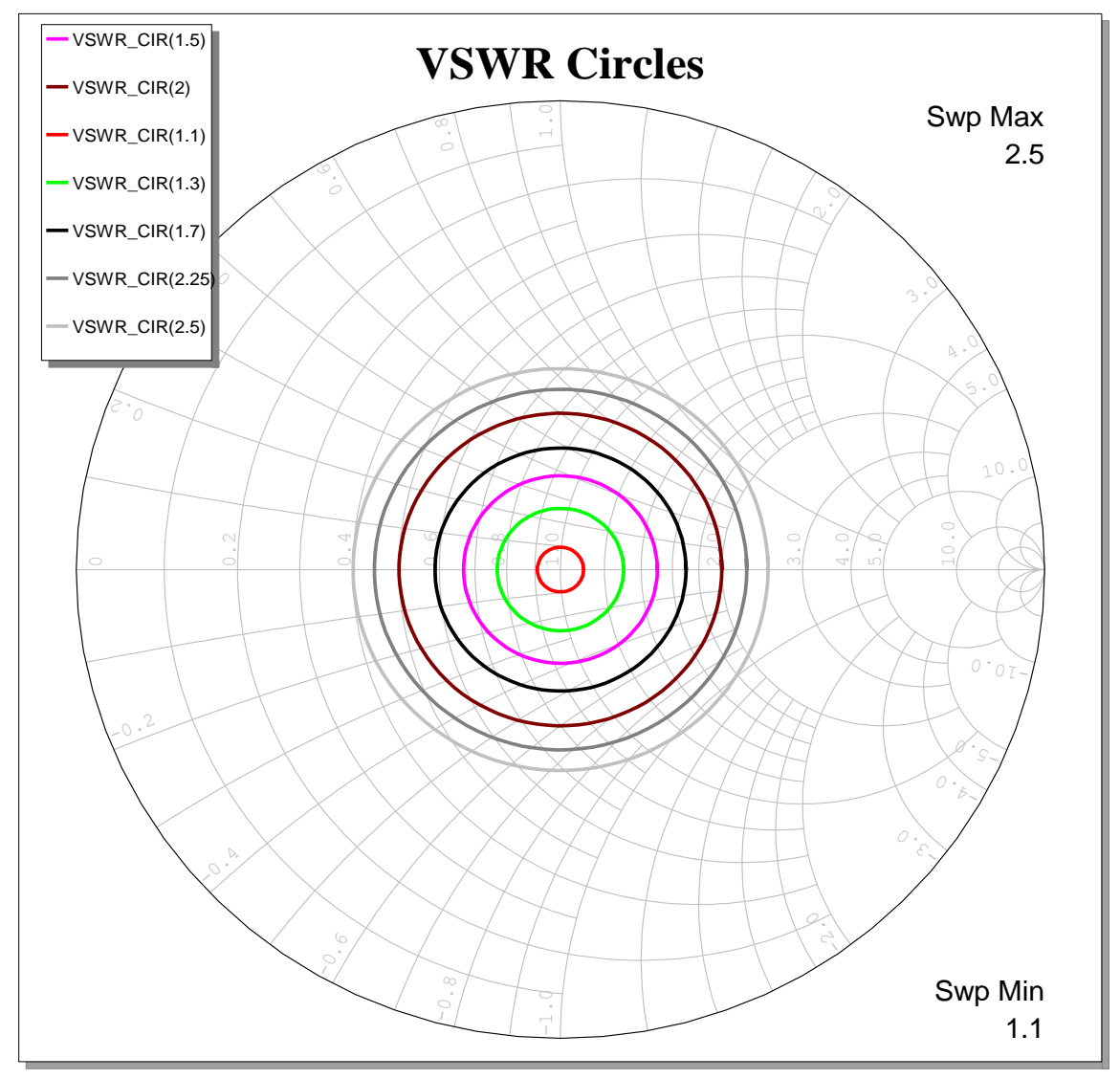

Fig. 6. Selected VSWRs. 


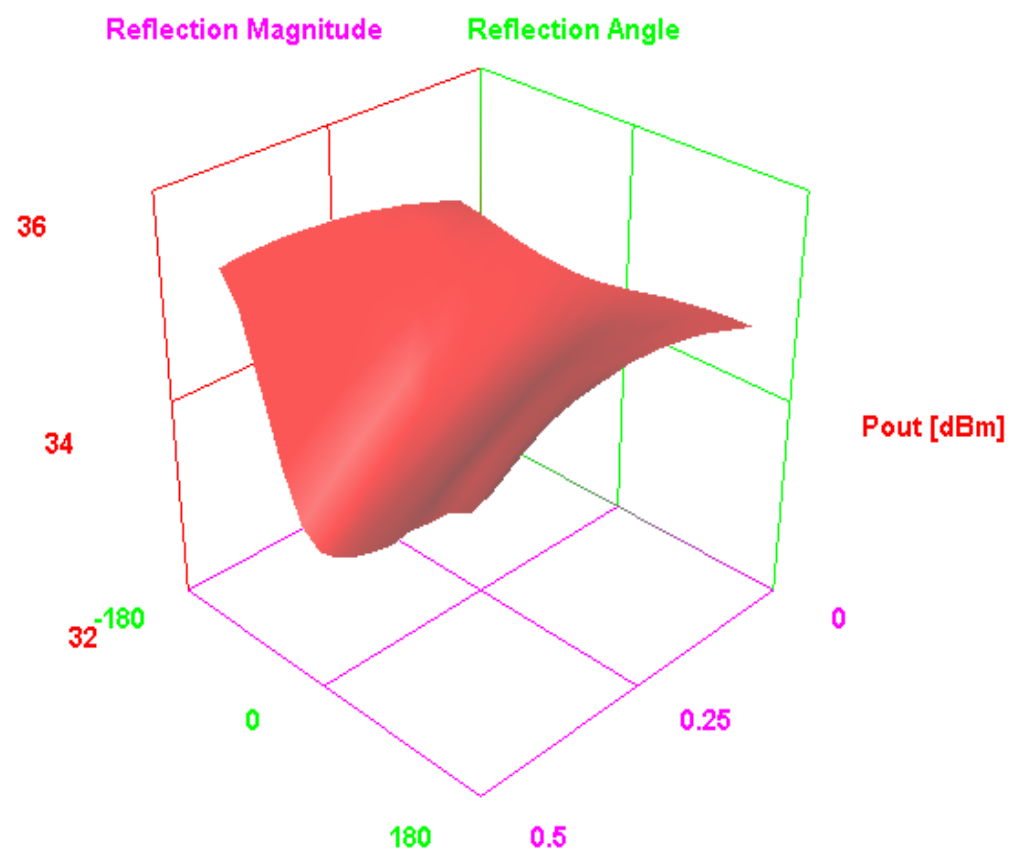

Fig. 7. Designed amplifier output power change for different VSWRs at the $8.5 \mathrm{~dB}$ back-off.

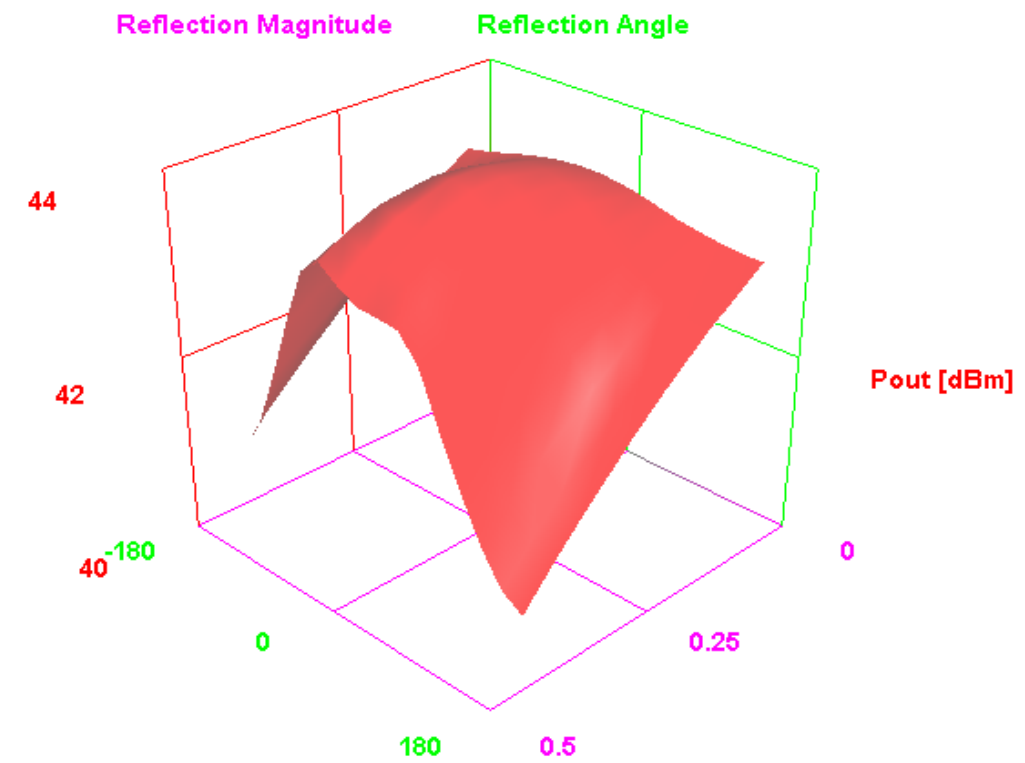

Fig. 8. Effect of different VSWRs on the output power at $43.2 \mathrm{dBm}$. 


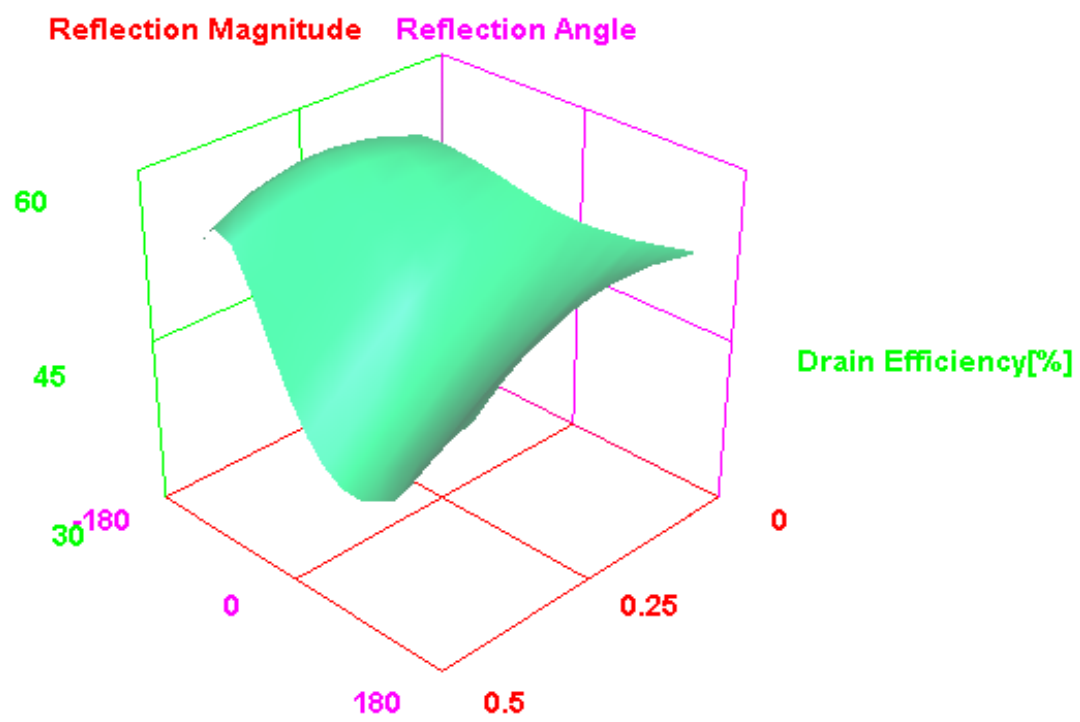

Fig. 9. The variation of drain efficiency for different VSWRs at $8.5 \mathrm{~dB}$ back-off.

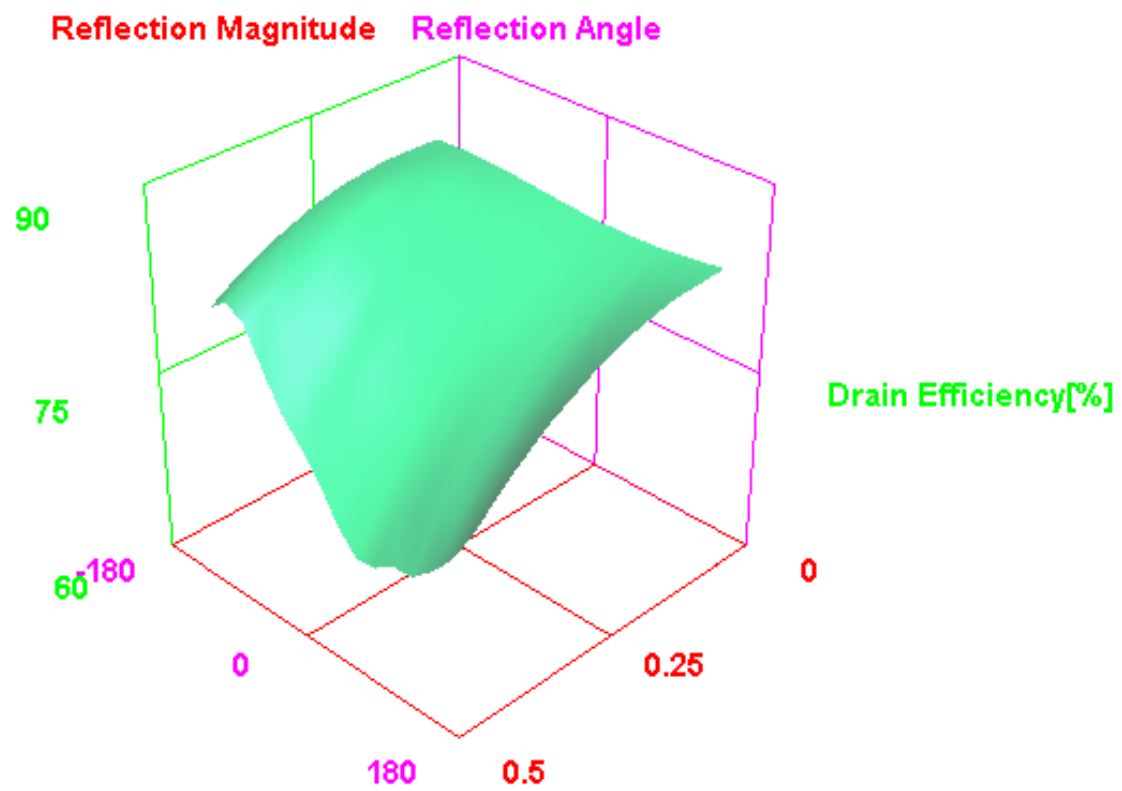

Fig. 10. The effect of different VSWRs on the drain efficiency for at $43.2 \mathrm{dBm}$. 


\section{Conclusions}

The three-way Doherty structure can be used for increasing the efficiency at the back-off region of the power amplifier. The designed three-way Doherty amplifier was tested when the amplifier is matched to $50 \Omega$ and a gain of $13 \mathrm{~dB}$ was obtained, a drain efficiency of $55 \%$ was achieved at $8.5 \mathrm{~dB}$ back-off power from the peak power of $43.2 \mathrm{dBm}$. The impedance changing at the output side can affect the drain efficiency and the output power of the amplifier. A 3.35 $\mathrm{dBm}$ variation of the output power was obtained for different VSWRs, moreover, the drain efficiency variation was about $20 \%$ for the same VSWRs. The structure of three-way Doherty amplifier or sequence of turning the peaking amplifiers on affect the amplifier performance for different VSWRs.

\section{Acknowledgements}

This paper is sponsored by the European Union's Horizon 2020 Research and Innovation program under grant agreement H2020-MSCA-ITN-2016-SECRET-722424.

\section{REFERENCES}

[1] B. Kim, Doherty Power Amplifiers: From Fundamentals to Advanced Design Methods. Elsevier Science, 2018.

[2] V. Camarchia, M. Pirola, R. Quaglia, S. Jee, Y. Cho, and B. Kim, "The Doherty Power Amplifier: Review of Recent Solutions and Trends," IEEE Transactions on Microwave Theory and Techniques, vol. 63, no. 2, pp. 559-571, 2015, doi: 10.1109/TMTT.2014.2387061.

[3] A. M. Abdulkhaleq et al., "Noise cancellation for compact MIMO systems," in the 5th International Conference on Internet Technologies and Applications, 2013. [Online]. Available: http://hdl.handle.net/10454/9585

[4] M. Sajedin, I. T. E. Elfergani, J. Rodriguez, R. Abd-Alhameed, and F. M. Barciela, "A Survey on RF and Microwave Doherty Power Amplifier for Mobile Handset Applications," Electronics, vol. 8, no. 6, 2019, doi: 10.3390/electronics8060717.

[5] A. M. Abdulkhaleq et al., "A 70-W Asymmetrical Doherty Power Amplifier for 5G Base Stations," in Broadband Communications, Networks, and Systems, Cham, V. Sucasas, G. Mantas, and S. Althunibat, Eds., 2019// 2019: Springer International Publishing, pp. 446-454.

[6] S. C. Cripps, RF Power Amplifiers for Wireless Communications (Artech House microwave library). Artech House, 2006.

[7] A. M. Abdulkhaleq et al., "Recent Developments of Dual-Band Doherty Power Amplifiers for Upcoming Mobile Communications Systems," Electronics, vol. 8, no. 6, 2019, doi: 10.3390/electronics8060638.

[8] A. M. Abdulkhaleq et al., "18-W Three-Way Doherty Amplifier For 5G Applications," presented at the Antennas and Propagation Conference 2019, Birmingham, UK, 2019.

[9] A. M. Abdulkhaleq et al., "Load-Modulation Technique for Next Generation Mobile," presented at the 2nd SECRET Workshop - Secure Network Coding for Reduced Energy next Generation Mobile Small Cells,IEEE 5G World Forum, Dresden, Germany, 2019.

[10] A. M. Abdulkhaleq et al., "Doherty Power Amplifier for LTE-Advanced Systems," Technologies, vol. 7, no. 3, 2019, doi: 10.3390/technologies7030060. 
[11] A. M. Abdulkhaleq et al., "A 70-W Asymmetrical Doherty Power Amplifier for 5G Base Stations," presented at the 9th International Conference on Broadband Communications, Networks, and Systems, Faro, Portugal, 2018.

[12] W. H. Doherty, "A new high-efficiency power amplifier for modulated waves," The Bell System Technical Journal, vol. 15, no. 3, pp. 469-475, 1936, doi: 10.1002/j.15387305.1936.tb03563.x.

[13] M. Liu, X. Fang, H. Huang, and S. Boumaiza, "Dual-band 3-way Doherty Power Amplifier with Extended Back-off Power and Bandwidth," IEEE Transactions on Circuits and Systems II: Express Briefs, pp. 1-1, 2019, doi: 10.1109/TCSII.2019.2907481.

[14] A. M. Abdulkhaleq et al., "Antennas Impedance Changing Effect Three-Way Doherty Amplifier for Green RF Communications," presented at the EuCAP 2020, 15-20March-2020, 2020.

[15] A. M. Abdulkhaleq et al., "A Compact Load-Modulation Amplifier for Improved Efficiency Next Generation Mobile," presented at the the 50th The European Microwave Conference (EuMC), The Jaarbeurs, The Netherlands, 2020.

[16] M. J. Roberts, "Understanding the 3 level Doherty," in 2016 46th European Microwave Conference (EuMC), 4-6 Oct. 2016 2016, pp. 1357-1361, doi: 10.1109/EuMC.2016.7824604. 